\title{
Semiconductor laser quantum noise limits
}

\section{Amnon Yariv, John E. Kitching, Yaakov Shevy}

Amnon Yariv, John E. Kitching, Yaakov Shevy, "Semiconductor laser quantum noise limits," Proc. SPIE 2378, Laser Frequency Stabilization and Noise Reduction, (26 April 1995); doi: 10.1117/12.208221

SPIE. Event: Photonics West '95, 1995, San Jose, CA, United States 


\title{
Semiconductor laser quantum noise limits
}

\author{
A. Yariv and J. Kitching \\ Department of Applied Physics, 128-95, \\ California Institute of Technology, Pasadena, CA 91125 \\ Y. Shevy \\ Department of Physics, University of Miami, P.O. Box 248046, Coral Gables, FL 33124
}

\begin{abstract}
The quantum mechanical limits to the fundamental noise performance of semiconductor lasers are reviewed. Recent advances in pushing the laser noise below these limits are then discussed with emphasis on pumpsuppression, electronic feedback and correlation techniques such as optical feedback. It is found that narrowlinewidth semiconductor lasers with sub-shot-noise photon statistics are within the reach of current technology.
\end{abstract}

\section{INTRODUCTION}

Since the earliest days of laser oscillators, issues of noise in these devices have been an important consideration from both a basic science viewpoint and the design aspect. Certain limits, such as the Schawlow-Townes linewidth ${ }^{1,2}$ and the shot noise limit were recognized early on as being fundamental to many types of lasers. Semiconductor lasers, in particular, have been useful devices for studying the quantum noise properties of lasers. Due to their small mode volume and low facet reflectivity, semiconductor lasers typically have linewidths of several megahertz which are dominated by fundamental noise sources such as spontaneous emission into the lasing mode. In addition, semiconductor laser cavities have relatively large cavity bandwidths with the result that the noise at frequencies of up to tens of $\mathrm{GHz}$ is determined mainly by the dynamics of the gain medium and its interaction with the optical field. Hence, semiconductor lasers have been a rich area for investigating the quantum limits to laser noise performance.

In recent years, advances in the understanding of these fundamental noise properties have pushed semiconductor laser performance beyond what was once thought to be basic limitations for both linewidth and amplitude noise. The discovery of the importance of phase-amplitude coupling in semiconductor lasers ${ }^{3,4}$ have led to useful schemes for reducing both the laser spectral linewidth $h^{5,6}$ and the amplitude noise. ${ }^{5,7,8}$ In addition, the understanding of the interaction of semiconductor lasers with their pumping circuits ${ }^{9,10}$ has given rise to amplitude squeezed light sources capable of generating light which, when photodetected, exhibits a photocurrent noise power below the shot noise level. The ease with which such sources can be built and the large squeezing bandwidths obtained make them an attractive tool for many applications. Other correlations between, for example, the junction 
voltage and the amplitude or phase noise, can also be used in conjunction with electronic feedback or feedforward to reduce the laser noise. ${ }^{11,12}$

Due to the ubiquity of semiconductor diode lasers in industrial settings, applications of low-noise devices are many and varied. A worldwide optical communications network will require narrow-linewidth, stable semiconductor lasers locked to frequency standards at $1.55 \mu \mathrm{m}$. Electronic and optical feedback systems are potentially important here to lock semiconductor lasers to atomic transitions. Atomic physicists are now turning to semiconductor lasers to replace large and expensive dye lasers currently used in many atomic physics experiments. ${ }^{13}$ Optical feedback techniques are beginning to be used to generate the narrow linewidths and tunability required for experiments such as laser cooling and trapping of atoms. Portable, cheap frequency standards have been proposed using atoms trapped with semiconductor lasers. ${ }^{14}$ Amplitude squeezed light may find use in certain precision measurements such as gravitational-wave detection ${ }^{15}$ in which the shot noise is a crucial barrier to the observation of weak signals. In addition, optical communication schemes have been devised to take advantage of the noise redistribution which occurs in amplitude-squeezed states in order to either increase transmission rates ${ }^{16}$ or secure transmission secrecy. ${ }^{17}$

This paper first reviews the fundamental limits to noise and its reduction in semiconductor lasers. Phase diffusion and the Schawlow-Townes linewidth are discussed as well as the basic origins of amplitude noise and the effects of phase-to-amplitude coupling on the laser noise. Certain excess noise sources, such as $1 / \mathrm{f}$ noise, are also briefly mentioned insofar as they relate to limiting the ultimate results obtainable with some noise reduction techniques. Methods for reducing the noise below these limits including pump-suppression, optical feedback and electronic feedback are then examined. Finally, some conclusions concerning the ultimate obtainable noise performance are drawn, evaluating the potential for current technology to obtain these goals.

\section{FREQUENCY NOISE AND LINEWIDTH REDUCTION IN SEMICONDUCTOR LASERS}

\subsection{Semiconductor laser linewidth}

An expression for the spectral linewidth of a laser oscillator was first derived by Schawlow and Townes ${ }^{1,2}$ using thermodynamic properties of optical waveguides and resonant cavities. Their results were later shown ${ }^{18}$ to be valid only for lasers below threshold since their noise analysis did not include the gain clamping mechanism responsible for suppressing the amplitude noise above threshold.

A somewhat more intuitive way of understanding the effects of spontaneous emission, for example, on the spectral properties of the laser's optical field is through the phasor model shown in Figure 1. In this model, the optical field is represented by a phasor of length $\sqrt{\bar{n}}$, where $\bar{n}$ is the number of photons in the lasing mode, which rotates in the complex plain at the oscillation frequency, $\omega$. Fluctuations in the optical field due to spontaneous emission are accounted for by the random addition of vectors with length unity and random phase to the end of the field phasor. Such events occur at random times but with a mean rate equal to the rate of spontaneous emission into the mode and cause changes in both the length of the phasor (field amplitude) and its phase.

Fluctuations in the field amplitude are strongly damped above threshold, however, because gain clamping produces a strong restoring force for the optical field intensity. The field phase, on the other hand, sees no such restoring force and is free to execute a random walk in time. The mean square fluctuation in the phase of the optical field after $N$ spontaneous emission events occurring in time $t$ can therefore be calculated to be ${ }^{19}$

$$
\left\langle[\Delta \theta(N)]^{2}\right\rangle=\frac{1}{\bar{n}}\left\langle\cos ^{2}(\phi)\right\rangle N=\frac{1}{2 \bar{n}} \frac{\mu t}{t_{c}}
$$




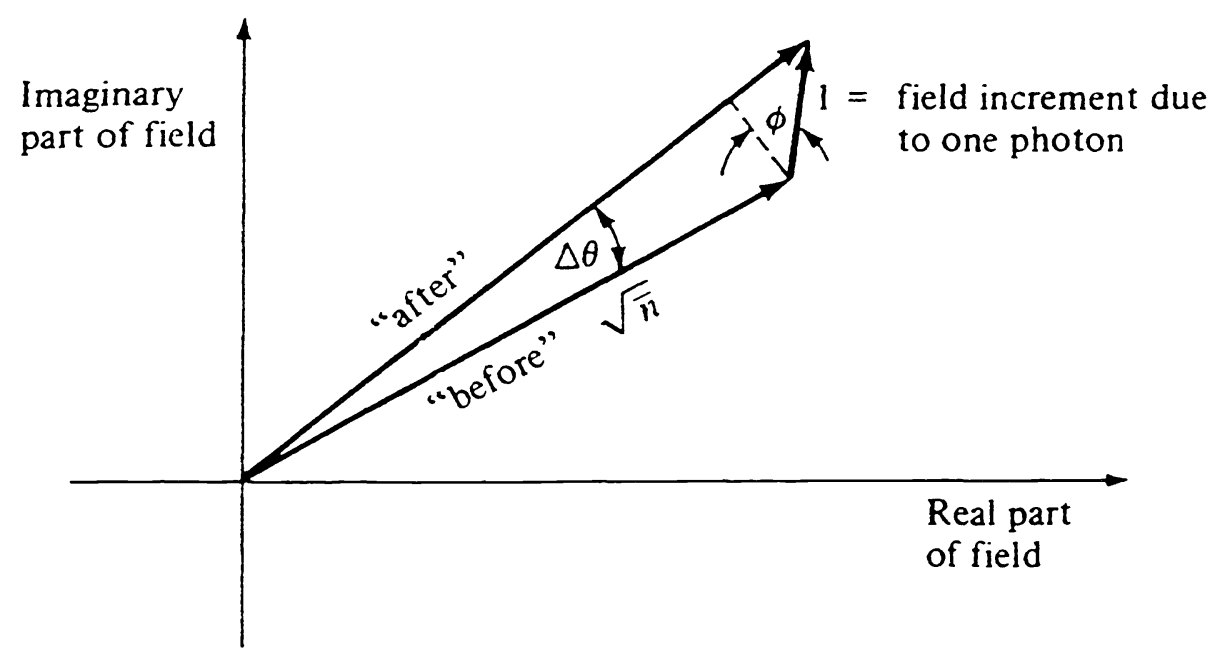

Figure 1: Phasor model for the effect of a single spontaneous emission event on the laser field (after Ref. [19]).

where $\mu=N_{2} /\left(N_{2}-N_{1}\right)$ is the inversion parameter and $t_{c}$ is the photon lifetime of the cold laser cavity. Under the assumption that the observation time is much longer that the time between the uncorrelated spontaneous emission events, the possible values of $\left\langle\Delta \theta^{2}\right\rangle$ are Gaussian distributed and the field spectrum can be shown to be Lorentzian with a FWHM

$$
\Delta \nu=\frac{\mu}{4 \pi \bar{n} t_{c}}=\frac{2 \pi h \nu\left(\Delta \nu_{1 / 2}\right)^{2} \mu}{P}
$$

where $\Delta_{1 / 1 / 2}$ is the cold cavity linewidth and $P$ is the output power. For a typical semiconductor laser this linewidth is a few megahertz at milliwatt output powers. Thus, unlike most gas or solid state lasers where mirror vibrations and other technical noise sources obscure the quantum linewidth, a semiconductor laser's linewidth is dominated by the quantum mechanical process of spontaneous emission.

While the phasor model of laser frequency noise accounts for the gross features of the noise behavior, it is incomplete in two ways. The first is that it is clearly a semi-classical theory: it does not treat the optical field as a quantum mechanical variable and therefore noise due to the discrete nature of light is not present. However, at frequencies below the cavity bandwidth the phase diffusion process usually contributes noise far in excess of the standard quantum limit (SQL) and therefore the quantum properties of the light can usually be ignored in this region. It should be noted, as will be described in Section 4, that the Heisenberg Uncertainty Principle which gives rise to the SQL does indeed generate the ultimate limit to linewidth reduction schemes and must be taken into account when determining the minimum obtainable linewidths. Secondly, there is the issue of phase-to-amplitude coupling which occurs in semiconductor lasers as a result of the asymmetrical gain profile and non-zero resonant refractive index at the lasing wavelength. This effect will be discussed in the following section.

\subsection{Phase-amplitude coupling}

The first measurements of the spectral linewidth of semiconductor lasers ${ }^{21}$ resulted in values 50 times larger than that predicted by the Schawlow-Townes expression, (2). Three years later, an explanation was proposed ${ }^{3,4}$ : that the asymmetrical gain profile in a semiconductor laser could result in a large coupling of amplitude noise into the field phase generating excess phase noise and hence an increased linewidth.

The essence of the phase-amplitude coupling argument is shown in Figure 2. For a typical gas or solid state 

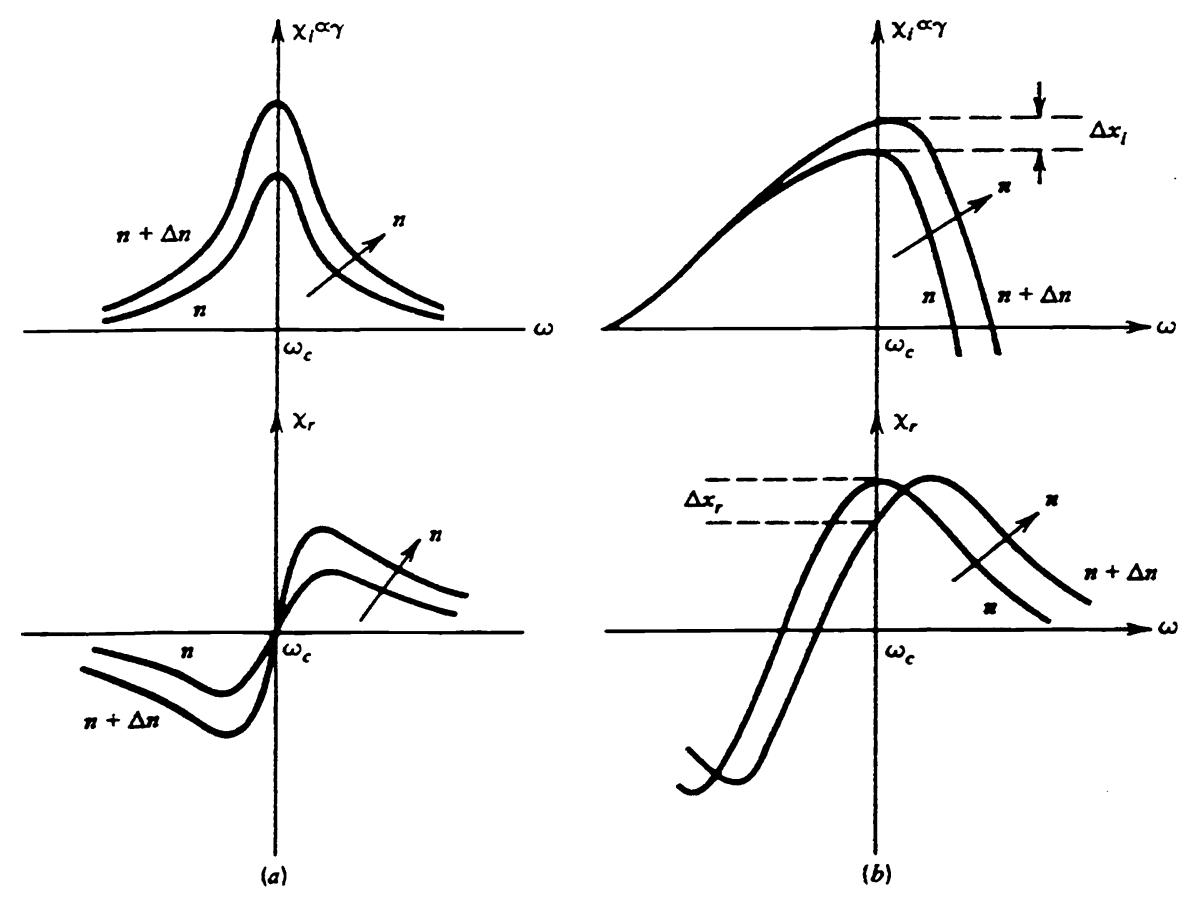

Figure 2: The gain $\left(\chi_{i}\right)$ and dispersion $\left(\chi_{r}\right)$ curves in (a) typical solid state lasers and (b) semiconductor lasers (after Ref. [20]).

laser (see Figure 2(a)), the gain profile and resonant refractive index are symmetrical as a function of frequency about their center frequencies. If the lasing frequency is close to the gain peak, fluctuations in the gain do not cause any substantial change in the refractive index seen by the internal optical field. For a semiconductor laser, however, the situation is very different. The gain profile in a semiconductor laser is asymmetrical, its shape produced at the low frequency end by the density of carrier states and at the high frequency end by the Fermi function cutoffs. Hence, as can be seen in Figure 2(b), the resonant refractive index at the gain peak is non-zero (the oscillator is detuned) and changes with the gain as required by the Kramers-Kronig relations. Fluctuations in the gain, caused by spontaneous emission events into the lasing mode, are therefore coupled into the field phase resulting in excess phase noise.

This phase-amplitude coupling is described quantitatively by the linewidth enhancement factor or $\alpha$-parameter defined by

$$
\alpha=\frac{\partial \chi_{r} / \partial N_{c}}{\partial \chi_{i} / \partial N_{c}}
$$

where $\chi_{r}$ and $\chi_{i}$ are the real and imaginary parts of the optical susceptibility of the lasing medium and $N_{c}$ is the carrier density. The numerical value of $\alpha$ depends to some extent on the type of laser but is typically 4-6 for bulk lasers $^{22}$ and about half that for quantum well lasers ${ }^{23}$ as a result of the larger differential gain in these structures. The existence of phase-amplitude coupling in semiconductor lasers leads to several important consequences. One of these is that the excess phase noise generated because of the coupling results in an enhancement of the spectral linewidth of the laser by a factor of $1+\alpha^{2}$ which explains why measured linewidths of Fleming and Mooradian ${ }^{21}$ were substantially larger than those predicted by the phasor model. The unity term in $1+\alpha^{2}$ is due to the original phase fluctuations while the $\alpha^{2}$ term is caused by the added noise due to the phase-amplitude coupling. Another consequence of phase-amplitude coupling is a correlation between the amplitude and phase fluctuations of the laser. A semiclassical theory shows that the magnitude of this correlation at low frequencies, $\left|P_{\Delta r \Delta \psi}(\Omega=0)\right|$, is equal to $\alpha / \sqrt{1+\alpha^{2}}$ where $\left|P_{\Delta r \Delta \psi^{\psi}}(\Omega=0)\right|=1$ represents a perfect correlation. As $\alpha$ increases, the linewidth 
does likewise and the phase-amplitude correlation gets increasingly closer to unity as the fraction of phase noise uncorrelated with the field amplitude gets smaller.

The fully quantum mechanical theory which includes not only noise due to dipole moment fluctuations and spontaneous emission into the lasing mode but noise due to spontaneous emission into non-lasing modes predicts a correlation of ${ }^{11,24}$

$$
\left|P_{\Delta r \Delta \psi}(\Omega=0)\right|=\frac{\alpha}{\sqrt{\left(1+\alpha^{2}\right)(1+R / 2)}}
$$

where $R=i_{L} / i_{t h}-1$ is the pump rate, $i_{L}$ the injection current and $i_{t h}$ the threshold current of the laser. The additional term $R / 2$ is due to the effect of spontaneous emission into non-lasing modes. This noise component produces amplitude noise without a corresponding correlated component in the phase noise. Since this noise source dominates the laser amplitude noise at high pump rates (see Section 3.1), the amplitude-phase correlation decreases to zero as the injection current is increased far above threshold.

\subsection{Linewidth reduction}

Most methods for linewidth reduction in semiconductor fall into one (or both) of two classes. The first, weak optical feedback (see Figure 3), involves the reflection of a small fraction of the light output back into the laser after propagation through some external dispersive element. Using this method, reductions in the linewidth by up to four orders of magnitude have been obtained. ${ }^{5,6,25,26}$ The second method (see Figure 6) is to measure the frequency noise directly using a frequency discriminator and then to correct for the frequency fluctuations by electronically feeding the error signal back into the laser injection current.

\subsubsection{Optical feedback and dispersive loss}

Narrow linewidths were first produced from a semiconductor laser by operating it in an extended cavity configuration. ${ }^{27,28}$ In these experiments, one of the laser facets was anti-reflection coated and an external, highreflectivity mirror positioned in front of the facet to reflect the output light back into the laser. The idea here was to increase the laser cavity mode volume and thus the $Q$, essentially making it like a conventional gas or solid-state laser with a semiconductor providing the gain. Linewidths of around $10 \mathrm{kHz}$ were obtained in this fashion.

Weak optical feedback has been discussed as a method of reducing the linewidth of semiconductors since as early as 1980 when Lang and Kobayashi ${ }^{29}$ performed the first theoretical analysis of the subject. Several other theoretical papers ${ }^{7,30-35}$ have been written since then emphasizing different aspects of the problem. The basic mechanism for the linewidth reduction is illustrated in Figure 3. The figure shows a semiconductor laser with weak feedback from an external cavity which may contain some dispersive element such as a Fabry-Perot resonator or atomic vapor. The feedback field has some phase, $\phi_{1}$, with respect to the internal field of the laser which depends on the frequency of the laser because of the phase delay induced by the feedback field's trip through the external cavity. Due to the presence of the feedback field, the internal laser field sees a slightly altered complex reflectivity at the laser facet which depends on the oscillation frequency. This frequency dependent loss has been modeled by substituting into the equation of motion for the optical field, a frequency dependent photon lifetime which accounts for the frequency dependence of the facet loss to first order in the frequency deviation. The photon lifetime is thus written

$$
\frac{1}{\tau_{p h}}=\frac{1}{\tau_{p h}^{(0)}}+2 C \dot{\phi}(t)
$$

where $\tau_{p h}^{(0)}$ is the original (frequency independent) photon lifetime, $\dot{\phi}(t)$ is the instantaneous frequency deviation of the internal optical field and $C$ is a (possibly complex) constant. The real part of $C$ represents a change in the 
loss rate caused by the presence of the optical field and the imaginary part represents a change in the round-trip phase shift. The relative magnitudes of $C_{r}$ and $C_{i}$ depend on the steady state phase deviation between the internal field and the feedback field as will be discussed in more detail below.

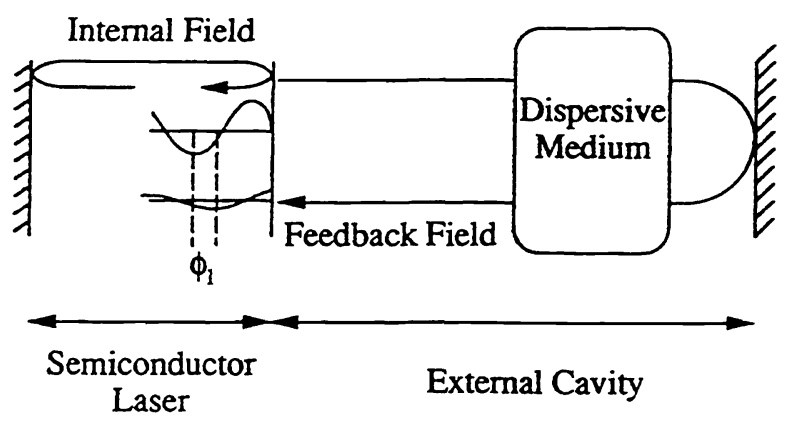

Figure 3: Schematic of optical feedback experiments.

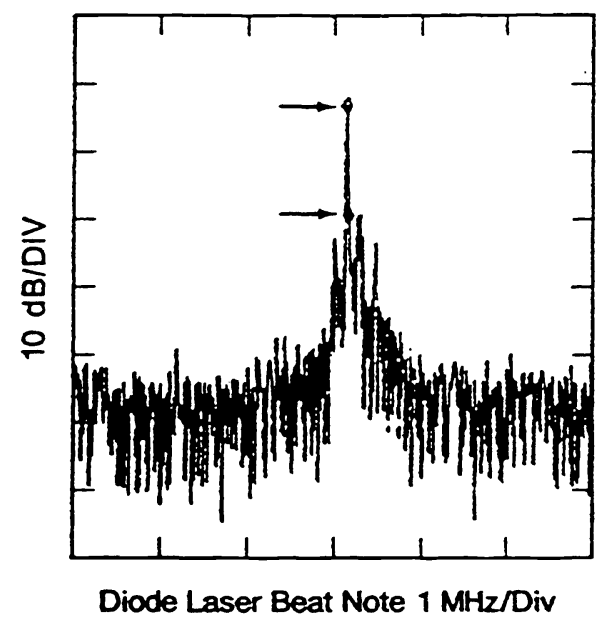

Figure 4: Diode laser beatnote with a bandwidth of approximately $20 \mathrm{kHz}$ (after Ref. [5]).

The frequency noise of the laser can be now affected in two ways. The first way is direct compensation of frequency fluctuation through the feedback-induced phase shift of the internal field, represented by $C_{i}$. The second way is through the phase-amplitude coupling mechanism. The original frequency fluctuation $\Delta \omega$ produces a change in the cavity loss rate, equal to $2 C_{r} \Delta \omega$, which causes a small change in the internal field intensity and in the gain (since the gain=loss condition must be satisfied). Accompanying this change in gain is a change in the refractive index and hence a change in the oscillation frequency. If this change is in the opposite direction to the original fluctuation, negative feedback results, thereby reducing the frequency noise and spectral linewidth. The linewidth reduction via this path clearly relies on phase-amplitude coupling and hence depends on the $\alpha$ parameter. The final result is that the linewidth under feedback conditions is given by ${ }^{7,36}$

$$
\Delta \nu=\Delta \nu_{0} \frac{1}{\left(1+C_{i}+\alpha C_{r}\right)^{2}}
$$

where $\Delta \nu_{0}$ is the linewidth under free-running conditions. It is clear from (6) that not only can the original enhancement of the linewidth caused by the phase-amplitude coupling be eliminated but that the linewidth can in fact be brought far below the original Schawlow-Townes limit if $C$ is made large enough. It can also be seen that for the same coupling strength $C$, the noise reduction due to the phase-amplitude coupling mechanism can be substantially larger than the equivalent reduction from the direct compensation due to the factor of $\alpha$ multiplying the $C_{r}$ term in (6).

It remains to determine how the parameters $C_{r}$ and $C_{i}$ depend on the feedback power, $P_{f b}$, and phase $\phi_{0}=\omega_{0} \tau+\phi_{m}$ where $\omega_{0}$ is the steady-state oscillation frequency, $\phi_{m}$ is the phase shift at the external cavity mirror and $\tau=\tau_{0}+\partial \phi / \partial \omega$ is the propagation delay through the external cavity which includes not only the empty cavity delay $\tau_{0}$ but also the group delay to the dispersive element $\partial \phi / \partial \omega$. These can be shown to be given by $^{36}$

$$
\begin{aligned}
& C_{r}=-\kappa\left(\omega_{0}\right) \tau \sin \left(\phi_{0}\right)+\left.\frac{\partial \kappa}{\partial \omega}\right|_{\omega=\omega_{0}} \cos \left(\phi_{0}\right) \\
& C_{i}=\kappa\left(\omega_{0}\right) \tau \cos \left(\phi_{0}\right)+\left.\frac{\partial \kappa}{\partial \omega}\right|_{\omega=\omega_{0}} \sin \left(\phi_{0}\right)
\end{aligned}
$$


where $\kappa(\omega)=\left(1-r_{c}\right)^{2} /\left(r_{c} \tau_{c}\right) \sqrt{P_{f b}(\omega) / P_{\text {out }}}$ is the feedback coupling rate, $r_{c}$ is the facet field reflectivity, $\tau_{c}$ the laser cavity round-trip delay time and where we have allowed the feedback power to be a function of frequency in case the laser is not tuned to the peak of the resonant element. In the case of $\partial \kappa / \partial \omega=0$, the relative strengths of $C_{r}$ and $C_{i}$ depend on the feedback phase $\phi_{1}=\phi_{0} \bmod 2 \pi$. If the feedback field is exactly in phase with the internal field $\left(\phi_{1}=0\right)$ then small changes in the phase of the feedback field generate changes in only the phase of the internal field and the corresponding noise reduction depends only on $C_{i}\left(C_{r}=0\right)$. If, on the other hand, the feedback field is $\pi / 2$ out of phase with the internal field $\left(\phi_{1}=\pi / 2\right)$, then changes in feedback field phase cause changes only in the amplitude of the internal field and the noise reduction depends only on the amplitude-phase coupling and $C_{r}\left(C_{i}=0\right)$.

Experimental verification of linewidth reduction has been performed by a number of groups. The first to accomplish a large linewidth reduction using weak feedback were the Favre et. al. ${ }^{37}$ who measured linewidth reductions by over two orders of magnitude using weak feedback from a single-mode fiber cavity. Dahmani, Hollberg and Drullinger ${ }^{5}$ have obtained a linewidth of $20 \mathrm{kHz}$ using feedback from a Fabry-Perot optical cavity as shown in Figure 4. The large dispersion of the optical cavity increased the value of $C$ and therefore enhanced the linewidth reduction. In addition, the cavity served to stabilize the center frequency of the laser. Similar feedback schemes have also been implemented using feedback from cavities incorporating atomic vapors. ${ }^{6,38} \mathrm{~A}$ linewidth of $10 \mathrm{kHz}$ was obtained by the authors ${ }^{6}$ using feedback from a velocity-selective transition in Cs vapor. Atomic resonance schemes have the added advantage over cavity feedback that the resonance frequency does not drift in time and therefore excellent absolute frequency stability can be obtained. ${ }^{26}$

\subsubsection{Low frequency stability and $1 /$ f noise}

Three unexpected features of narrow-linewidth lasers with optical feedback have been found in certain optical feedback experiments. ${ }^{6,39,25}$ First, the lineshape of the laser field spectrum under feedback conditions is found agree much better to a Lorentzian to the power $3 / 2$ fit rather than a Lorentzian fit (Figure $5(\mathrm{a})$ ). Second, the inverse linewidth is found to depend linearly on $\left(1+C_{i}+\alpha C_{r}\right)$ rather than going as the square as predicted by Eq. (6) (See Figure 5(b): here $P=1+C_{i}+\alpha C_{r}$ ). Finally, for typical values of $C$ generated in these experiments ${ }^{6}$ (roughly 1000), the obtained linewidth reduction is smaller by several orders of magnitude than the theoretically predicted value $\left(10^{6}\right)$.

These results can be explained by the existence of $1 / \mathrm{f}$ noise at low frequencies in the frequency noise power spectrum of the laser. ${ }^{40,25,39}$ The $1 / \mathrm{f}$ noise becomes important at linewidths below roughly $1 \mathrm{MHz}$ when the linewidth is dominated by the effects of the low-frequency part of the noise spectrum. It can be shown that for $1 / \mathrm{f}$ noise, the laser linewidth is proportional to the square-root of the frequency noise power rather than being proportional to the noise power itself as is the case for white noise ${ }^{40}$ Therefore in this regime, while the optical feedback still reduces the frequency noise power spectrum by a factor of $\left(1+C_{i}+\alpha C_{r}\right)^{2}$, the linewidth is reduced by only the square-root of this quantity. This behavior can be understood intuitively by considering that as the frequency noise power spectrum is reduced, a lower frequency portion of the spectrum is responsible for generating the linewidth since the linewidth itself becomes smaller. While the white noise level is of course independent of frequency, the $1 / \mathrm{f}$ noise level increases with decreasing frequency making the reduction in the linewidth smaller.

These predictions have been verified by an experiment ${ }^{39}$ which examined the transition from white noise dominance of the linewidth to $1 / \mathrm{f}$ noise dominance with increasing feedback level. At low feedback levels, the inverse linewidth was found to go as $\left(1+C_{i}+\alpha C_{r}\right)^{2}$ while at higher feedback levels, where the linewidth was narrower and $1 / \mathrm{f}$ noise more important, a linear dependence was measured. $1 / \mathrm{f}$ noise, therefore, becomes the limiting factor in determining the spectral linewidth of the laser under optical feedback conditions and in this case the linewidth is less sensitive to the feedback than a linewidth generated by white noise would be. However, due to the low frequencies at which the noise occurs, it becomes easier to reduce using electronic feedback than is the higher frequency white noise component. 


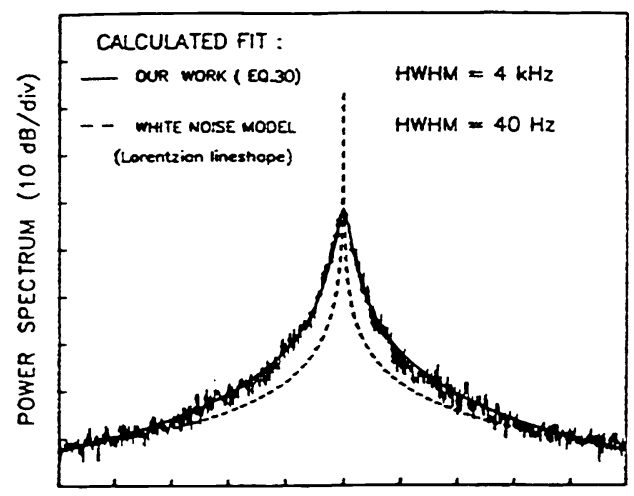

FREQUENCY AXIS ( $50 \mathrm{kHz} / \mathrm{div}$ )

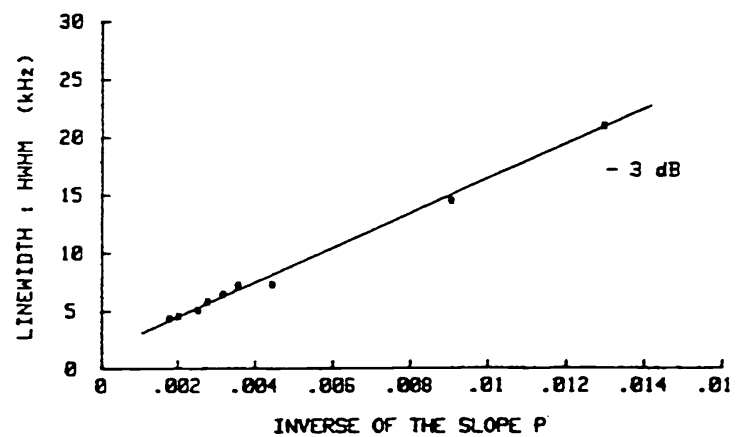

(b)

Figure 5: Anomalous results found in experiments on linewidth reduction with optical feedback: (a) The field spectrum lineshape is found to be a Lorentzian to the power $3 / 2$ (solid curve) rather than a Lorentzian (dashed curve) and (b) the inverse linewidth is found to be proportional to $Q=P=1+C_{i}+\alpha C_{r}$ (after Ref. [25]).

\subsubsection{Electronic feedback}

Another popular technique for reducing the frequency noise in semiconductor lasers is the use of electronic feedback. The principle here is simple and is shown in Fig. 6(b). The frequency of the laser is first compared to that of a fixed reference such as an external Fabry-Perot cavity or atomic transition. The difference between the two frequencies is then translated into an electrical signal and fed back into the laser injection current which in turn changes the laser frequency to bring it closer to the reference.

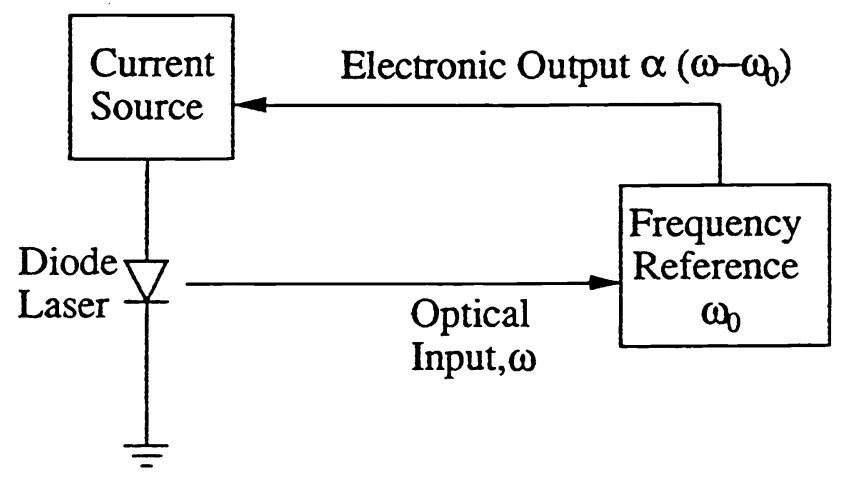

Figure 6: Schematic of electronic feedback experiments.

Several methods for translating the frequency difference into a voltage have been proposed, two of the more popular being the FM sideband locking technique ${ }^{41,42}$ and direct discrimination using the side of the resonance line. In the direct detection technique, the laser is tuned to the side of the resonance line and the reflected (or transmitted) power is measured with a photodetector. Frequency fluctuations are translated into intensity fluctuations by the local frequency dependence of the reflection (or transmission) coefficient which results in photocurrent fluctuations which can be amplified and sent directly back to the laser injection current driver. 


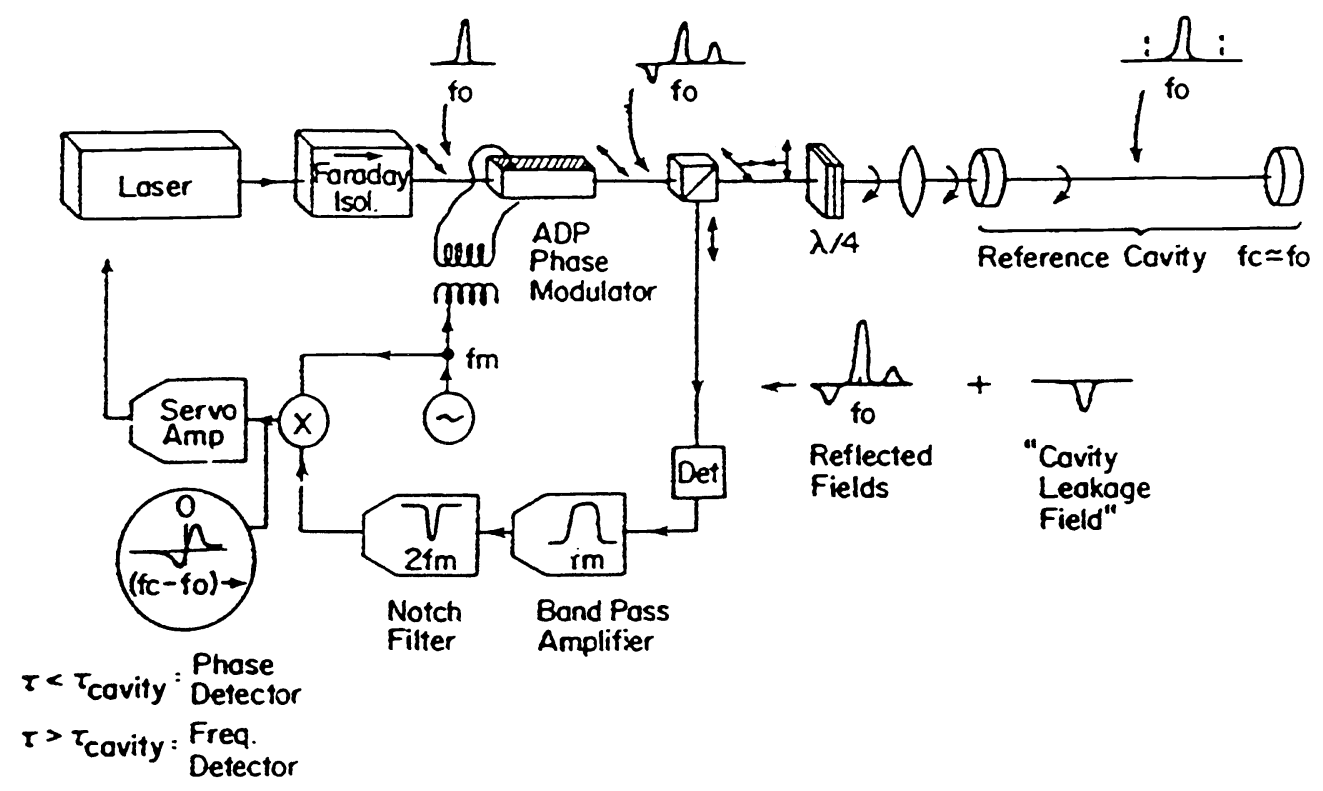

Figure 7: FM sideband locking technique for frequency stabilization (after Ref. [42]).

While this technique has the advantage of being simple to implement, it is unable to lock the laser to the peak of the resonance where the slope is zero. The amplitude noise of the laser is also a problem since this noise will be picked up by the detector and translated into frequency fluctuations by the feedback.

FM sideband locking avoids both of the above problems (see Figure 7). In this technique, the phase of the laser optical field is modulated at a frequency roughly equal to the width of the resonant element, generating frequency sidebands in the field spectrum of the laser. The optical field is then passed through the resonant element and sent into photodetector. When the laser frequency is exactly equal to the peak of the reference resonance, the sidebands suffer equal attenuation and phase delay and no beatnote at the modulation frequency is produced in the resulting photocurrent. If the laser frequency is slightly off-center, however, the asymmetry in the sideband transmission produces a beatnote at the modulation frequency. The detector current is then demodulated with an RF mixer resulting in a DC output signal which has a large slope in the vicinity of the line center. This output can then be amplified and returned to the current supply to reduce the frequency noise of the laser. In addition to providing a way to lock the laser to the top of the resonance lineshape, the FM sideband locking method provides superior signal-to-noise to the direct detection method. The reason is that intensity fluctuations of the laser create common-mode noise in the detection system which is rejected by demodulation. As a result, the basic noise limitation to the FM sideband technique is the shot noise limit. ${ }^{42}$

It should be noted that there is nothing which prevents electronic feedback schemes from being implemented simlutaneously with optical feedback. In many ways they are complimentary techniques, the optical feedback being effective at reducing the higher frequency noise where electronic feedback is harder to use and the electronic feedback providing the additional noise control at lower frequencies where excess noise such as $1 / \mathrm{f}$ noise is often found.

There have been many experimental results over the years which have locked semiconductor lasers to external cavities or atomic resonances. We concentrate here on more recent results which have generated narrow linewidths in addition to low-frequency stability. Shin and Ohtsu ${ }^{43}$ have produced a linewidth of $7 \mathrm{~Hz}$ from a Fabry-Perot semiconductor laser at $830 \mathrm{~nm}$ using a combination of direct discrimination electronic feedback and optical feedback from an external cavity. The optical feedback reduced the linewidth to about $10 \mathrm{kHz}$ and the electronic feedback provided the remaining reduction. The frequency noise spectral density measured under optimum 


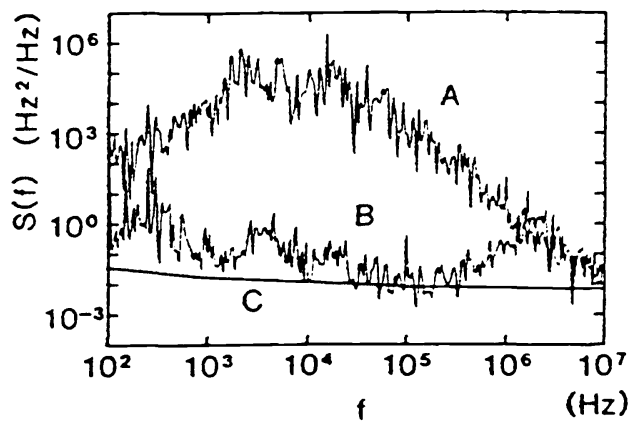

(a)

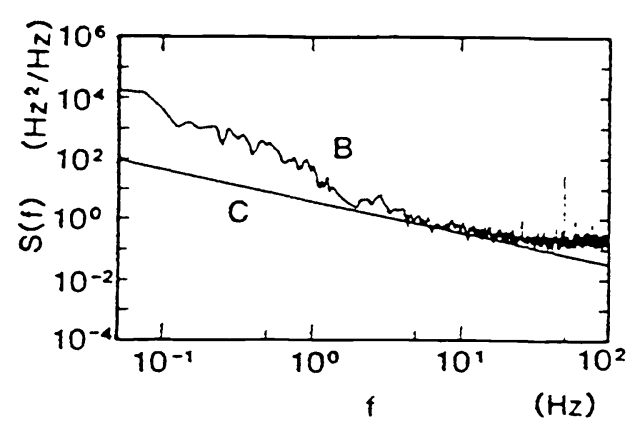

(b)

Figure 8: Frequency noise power spectral density at high frequencies (a) and low frequencies (b) from a semiconductor laser with optical and electronic feedback. Trace $\mathrm{A}$ is for a lower feedback gain and bandwidth than trace B. Trace $\mathrm{C}$ indicates the detection limit imposed by the diode intensity noise (after Ref. [43]).

conditions in shown in Fig. 8. A linewidth of $80 \mathrm{~Hz}$ has also been produced by electronic feedback alone in a multi-section DFB laser at $1.5 \mu \mathrm{m} .{ }^{44}$ Several experiments have concentrated on locking semiconductor lasers to atomic transitions. Double optical/electronic feedback systems have also been implemented using velocityselective transitions Cs vapour, for example. A spectral linewidth of $1.4 \mathrm{kHz}$ and an Allen variance of $2 \times 10^{-13}$ at an integration time of 1 second were obtained in this fashion. ${ }^{26}$

\section{AMPLITUDE NOISE IN SEMICONDUCTOR LASERS}

\subsection{The quantum theory of amplitude noise in semiconductor lasers}

While the phase noise and linewidth can be treated adequately within the semiclassical theory of the laser, a proper analysis of the low-frequency amplitude noise requires the use of a fully quantum mechanical optical field. The reason for this is that because of the strong gain clamping mechanism above threshold, the low-frequency amplitude noise of a semiconductor laser approaches the shot noise limit even at moderate pump rates. Since it is precisely the quantum nature of the field which gives rise to the SQL, the fully quantum mechanical theory of the laser must be used. While the low-frequency part of the spectrum is intrinsically non-classical, it should be noted that some amplitude noise phenomena such as the noise around the relaxation resonance peak can be calculated correctly within the semiclassical theory.

The intensity noise spectrum for the internal field of a laser was first calculated by $\mathrm{McCumber}^{45}$ using quantum mechanical rate equations with Langevin noise sources. His approach was later justified by $\mathrm{Lax}^{46}$ who used a shot noise analysis to obtain correlation functions for the McCumber's noise terms. McCumber's theory was first applied to semiconductor lasers by $\mathrm{Haug}^{9}$ who obtained equations of motion for the inversion and internal optical field photon number after the adiabatic elimination of the dipole moment variable. The next important advance came with the realization by Caves ${ }^{47}$ that the vacuum optical field incident on the front facet of the laser had to be included in the model to correctly describe the relation between the internal field of the laser and the external field emitted through the facet. Finally an understanding of the pumping mechanism in semiconductor lasers was added by Yamamot. ${ }^{10,48}$ to complete the model. This last step has led to the possibility of generating amplitude squeezed light from a semiconductor laser. 
The basic model can be described, after the adiabatic elimination of the dipole-moment operator, using two quantum Langevin equations for the inversion operator $N_{c}(t)$ and the internal optical field anihilation opeator $a(t)$ in addition to the input/output relation connecting the external field $r(t)$ with the internal field. These can be written ${ }^{10}$

$$
\begin{aligned}
& \frac{d}{d t} N_{c}(t)=P-\frac{N_{c}(t)}{\tau_{s p}}-\frac{\omega}{2 n^{2}} \chi_{i} a^{\dagger} a+\Gamma(t) \\
& \frac{d}{d t} a(t)=-\frac{1}{2}\left[\frac{1}{\tau_{p 0}}+\frac{1}{\tau_{p e}}+2 i\left(\omega-\omega_{0}\right)-\frac{\omega}{n^{2}}\left(\chi_{i}-i \chi_{r}\right)\right]+G(t)+\frac{f_{e}(t)}{\sqrt{\tau_{p e}}} \\
& r(t)=-f_{e}(t)+\frac{a(t)}{\sqrt{\tau_{p e}}}
\end{aligned}
$$

where $P$ is the pump rate, $\tau_{s p}$ is the spontaneous emission lifetime, $\omega$ is the laser oscillation frequency, $n$ is the non-resonant refractive index, $\chi\left(N_{c}\right)$ is the resonant optical susceptibility, $\tau_{p 0}$ and $\tau_{p e}$ are the cavity photon lifetimes due to internal absorption and mirror losses respectively and $\omega_{0}$ is the cold cavity resonant frequency. The Langevin noise term $\Gamma(t)$ drives the carrier variable and includes noise due to the pumping process, spontaneous emission into non-lasing modes and dipole moment fluctuations. $G(t)$ generates fluctuations in optical field variable and is due to dipole moment fluctuations and noise from internal optical losses. Finally, $f_{e}(t)$ is the vacuum field incident on the front facet of the laser and accounts explicitly for noise due to spontaneous emission into the lasing mode. The vacuum field $f_{e}(t)$ is also partially reflected off the front facet of the laser and therefore appears in the output coupling relation, (11).

The noise terms $\Gamma(t), G(t)$ and $f_{e}(t)$ have correlation functions ${ }^{10,49}$

$$
\begin{aligned}
& \langle\Gamma(t) \Gamma(u)\rangle=\delta(t-u)\left[P+\frac{N_{c 0}}{\tau_{s p}}+\left\langle E_{C V}\right\rangle\left(A_{0}^{2}+1\right)+\left\langle E_{V C}\right\rangle A_{0}^{2}\right] \\
& \left\langle G_{r}(t) G_{r}(u)\right\rangle=\left\langle G_{i}(t) G_{i}(u)\right\rangle=\delta(t-u) \frac{1}{4}\left[\frac{1}{\tau_{p 0}}+\left\langle E_{C V}\right\rangle+\left\langle E_{V C}\right\rangle\right] \\
& \left.\left\langle G_{r}(t) \Gamma(u)\right\rangle=-\delta(t-u) \frac{1}{2} A_{0}\left(\left\langle E_{C V}\right\rangle+\left\langle E_{V}\right\rangle\right\rangle\right) \\
& \left\langle G_{i}(t) \Gamma(u)\right\rangle=0 \\
& \left\langle f_{\epsilon r}(t) f_{e r}(u)\right\rangle=\left\langle f_{e i}(t) f_{e i}(u)\right\rangle=\frac{1}{4}
\end{aligned}
$$

where $\left\langle E_{C V}\right\rangle$ and $\left\langle E_{V C}\right\rangle$ are the mean rates of stimulated emission and absorbtion respectively. The terms in (12)-(16) can be understood by considering each as being composed of a large number of independent events, each producing an impulse change in the variables $a(t)$ and $N_{c}(t)$. In this case, the mean-square fluctuation of the variable is just equal to the mean rate of occurrence of the events multiplied by the mean-square change in the variable from each event. For the carrier noise, for example, the three ways in which the carrier density can change are through pumping, spontaneous emission or stimulated emission/absorption. Thus the correlation function $\langle\Gamma(t) \Gamma(u)\rangle$ is equal to a delta function (Markoffian processes are assumed) multiplied by the rates of occurrence for each process, $\mathrm{P}$ (for pump noise), $N_{c 0} / \tau_{s p}$ (for spontaneous emission) and $E_{C V}\left\langle a^{\dagger} a+1\right\rangle+E_{V C}\left\langle a^{\dagger} a\right\rangle$ (for stimulated emission/absorption) multiplied again by the effect of each event, which is to change the carrier density by unity. The cross-correlation functions include only those events which change,both quantities simultaneously. Poissonian pumping statistics have been assumed here and will be discussed further below.

Equations (9) and (10) can be solved by writing the carrier density and fields in terms of small signal quantities which fluctuate about mean values: $N_{c}(t)=N_{c 0}+\Delta N_{c}(t), a(t)=\left[A_{0}+\Delta A(t)\right] e^{i \Delta \phi(t)}$ and $r(t)=$ $\left[r_{0}+\Delta r(t)\right] e^{i \Delta \psi(t)}$. The Fourier transformed algebraic equations are then solved for each fluctuation variable $\Delta N_{c}(\Omega), \Delta A(\Omega)$ and $\tilde{\Delta \phi}(\Omega)$, the fluctuation of the external field $\tilde{\Delta r}(\Omega)$ is calculated using $(11)$ and the singlesided power spectral density of this quantity $P_{\Delta r}(\Omega)$ is found. In general, $P_{\Delta r}(\Omega)$ is a fairly complicated expression but it can be simplified when the noise frequency is much lower then the inverse of the stimulated emission lifetime of the carriers. In this case, the amplitude noise power spectral density of the external field, normalized to the 
shot noise limit of $2 P$, is given by

$$
P_{\Delta r}(0)=(1-\eta)+\eta\left[1+\frac{1}{R}+\frac{2}{\mu R^{2}}\right]
$$

where $\eta$ is the external differential quantum efficiency. The contributions from the different noise sources in this expression can be easily identified. Optical losses inside the laser are accounted for by $\eta$, pump noise by the unity term inside the brackets, spontaneous emission into non-lasing modes by the $1 / R$ term, dipole moment vacuum fluctuations (spontaneous emission into the lasing mode) by the last. It can be seen that far above threshold, if the internal losses are small compared to the facet losses $(\eta \approx 1)$, the noise is at the SQL and is determined by the pump noise only.

\subsection{Vacuum cancellation, pump suppression and amplitude squeezing}

We now discuss two aspects of the theory outlined in the previous section: the reason for which the vacuum field does not generate the SQL and methods for suppressing the pump noise. These two elements are keys to understanding how amplitude squeezed light is generated from a semiconductor laser. The absence of the vacuum field fluctuations at high pump rates is due to interference between the component of the vacuum field reflected from the front facet of the laser and the laser field itself, which of course contains the component transmitted through the facet. The pump noise is eliminated by driving the laser with a constant-current source.

As has been described by Yamamoto, ${ }^{10}$ it is the interference between the transmitted internal field and the reflected vacuum field which results in a complete cancellation of the noise due to $f_{e}(t)$ at high pump rates. This cancellation can be thought of in the following way. Imagine $f_{e}(t)$ to be not a vacuum field but an arbitrary classical optical field. This field incident on the laser facet causes the internal field of the laser to see an altered facet loss which is either smaller or larger than the original depending on the relative phase of the two fields. As a result, the gain (inversion) must also change to satisfy the gain=loss condition. But if the pump rate remains the same, this translates into an increased or decreased rate of stimulated emission into the mode. The amplitude of the internal field is therefore altered in a fashion which is correlated with the injected signal. In fact, when the simple calculation outlined above is carried out, one finds that the change in the portion of the internal field transmitted through the facet exactly cancels the portion of the injected field reflected from the facet. The laser, therefore, acts like a "matched termination" to an injected field: no reflections occur. Since this argument holds for an arbitrary weak optical field, it is certainly true for the vacuum field $f_{e}(t)$ as long as we consider only fluctuations which occur at frequencies well below the inverse of the carrier stimulated emission lifetime.

We now consider the noise due to pump fluctuations in more detail using arguments first proposed for semiconductor lasers by Yamamoto. ${ }^{4 \delta}$ There are three obvious ways to pump a semiconductor laser, each interacting with the carrier density in the active region in a different. way. The first is to pump the laser optically with classical light. In this case carriers are generated in a random fashion and therefore the pump current is indeed Poissonian and results in a shot noise limited external optical field as was found in (12). This is the type of pumping usually considered in laser models. ${ }^{9,50}$ A second way of pumping a semiconductor laser is with a voltage source. Since the junction voltage is proportional to the carrier density, this fixes the carrier density at a constant value. Fluctuations in the rate of stimulated emission, which are caused by the random nature of the emission process, cause corresponding fluctuations in the pump rate since, in order to keep the junction voltage constant, the carriers in the active region must be replaced as soon as they recombine to create a stimulated photon. Thus, in this case also, the pump current is Poissonian, the noise being caused by the random nature of the emission process rather than by fluctuations in the generation rate.

Finally, one can consider driving the laser with a constant current source or voltage source in series with a large resistor. In this case, fluctuations in the rate of stimulated emission cause the carrier density (junction voltage) to fluctuate and therefore change the gain in exactly the direction to compensate for the original fluctuation. If the rate of stimulated emission increases, the junction voltage decreases reducing the gain and therefore reducing the 
stimulated emission rate back towards its steady-state value. This "self-correcting" mechanism thereby reduces the pump fluctuations to zero in the limit of the current source series resistor being much larger than the diode differential resistance. ${ }^{48}$ The only pump noise that remains is the thermal noise in the source resistor which may be reduced to an arbitrarily small value by using a large enough resistance.

The frequency-dependence of the amplitude noise on the external field is shown in Figure 9 when the laser is pumped far above threshold. The noise from the external vacuum field is at the SQL at frequencies above the cavity bandwidth since the internal field of the laser cannot respond quickly enough to provide the canceling effect discussed above. At low frequencies, however, the vacuum fluctuations do cancel out and, under Poissonian pumping, the low frequency noise is dominated by the pump noise which generates the shot noise limit. But above the cavity bandwidth, the pump noise falls off due, again, to the inability of the internal field to communicate with the external field on short time scales. Thus, for Poissonian pumping, the total amplitude noise is a constant, at the SQL at all frequencies. When the laser is pump-suppressed by driving it with a constant current source, however, the pump noise is drastically reduced, generating large amplitude squeezing at frequencies well below the cavity bandwidth.

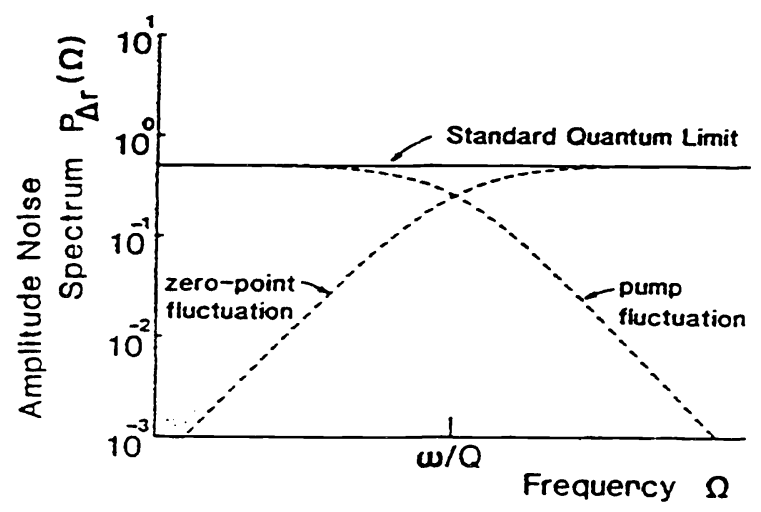

Figure 9: The amplitude noise on the external field, normalized to the SQL, for a semiconductor laser pumped far above threshold (after Ref. $[10])$.

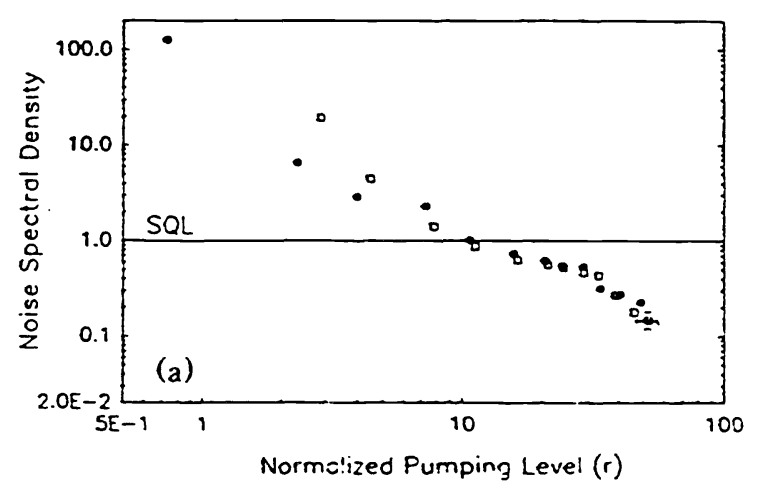

Figure 10: Measured amplitude noise powers as a function of pump rate (After Ref. [57])

The generation of squeezed light from a semiconductor laser has several significant advantages over other methods of producing squeezed light such as parametric downconversion ${ }^{51}$ and four-wave mixing. ${ }^{52}$ One is the relative ease with which the squeezing can be produced. Typical quadrature squeezing experiments require a table full of optics and complicated servo locking systems. ${ }^{51}$ The generation of amplitude squeezed light from a semiconductor laser requires only a resistor! It thus seems even feasible to manufacture amplitude squeezed light sources in a commercial setting. In addition the squeezing bandwidth for a semiconductor laser is essentially the cavity bandwidth if high enough pump rates can be achieved, ${ }^{53}$ which can be many GHz. In optical parametric oscillators, one of the most successful devices for generating quadrature-squeezed light, high finesse cavities are required to generate the high optical powers necessary for efficient downconversion thereby limiting the bandwidth significantly. Finally, semiconductor lasers generate "bright" squeezed light rather than squeezed vacuum: the squeezing sits on a large steady-state optical field, which is a useful property for many applications. 


\subsection{Experimental issues in the generation of amplitude squeezed light}

Several experimental groups have reported measuring amplitude squeezed light from a pump-suppressed semiconductor laser ${ }^{53-61,12}$ or LED. ${ }^{62}$ The amplitude noise is usually measured in one of two ways. The first is by directly detecting the light in a photodetector, measuring the photocurrent noise and comparing the measured noise level to that from a source known to be shot noise limited, usually a LED. The second method is through the use of a balanced homodyne detector. ${ }^{55,63}$ This method of detection has the advantage that the shot noise level can be internally calibrated by measuring the noise when the detector photocurrents are subtracted rather than added. The shot noise level can then be rechecked using LED's. Pump-suppression can be achieved either by placing a large resistor in series with a high voltage source or by replacing the resistor with an inductor which allows the DC current to pass unimpeded while suppressing the current noise at higher frequencies. Also, most experiments are carried out with the laser (and often the detectors) cooled to cryogenic temperatures in order to increase differential quantum efficiencies and to anable the pumping of the laser far above threshold. However several groups have now obtained squeezing from room temperature lasers and there are no fundamental reasons why such generation should be significantly less effective.

The largest degree of squeezing (of any kind) produced to date, $8.3 \mathrm{~dB}$ below the shot noise level, was reported by Yamamoto's group at NTT. ${ }^{57}$ They used cryogenically cooled lasers and direct detection of the light with LED calibration of the shot noise level. Their measured noise powers at frequencies between $50 \mathrm{MHz}$ and $150 \mathrm{MHz}$ are plotted versus the pump rate $R=i_{L} / i_{t h}-1$ in Figure 10. Squeezing is clearly obtained at pump rates above $R=10$. Interestingly, their minimum noise level is actually below what would be expected from an efficiency argument alone without any added noise from the laser itself. They explain this by assuming the existence of a non-lasing junction in parallel with the lasing junction and analyzing the current branching noise in the electronic circuit. They find that in this situation, current branching can reduce the external efficiency of the laser without affecting the noise adversely.

With the exception of the one result described above, all other measurements of amplitude squeezing in semiconductor lasers have turned up less than $4 \mathrm{~dB}$ of squeezing. Although poor current-to-current efficiencies are certainly one reason, the agreement between experiment and theory has not been particularly good either indicating that additional mechanisms not described by the basic theory may be generating excess noise in the laser. Foremost among these is possible excess noise caused by the existence of weak side-modes. ${ }^{57,60}$ In an inhomogeneously broadened medium such modes would have a noise level far in excess of their SQL (due to their being close to threshold) and thus, although they would not contribute much of the total power, their noise contribution could be significant. Another mechanism, asymmetrical cross-mode non-linear gain in an otherwise homogeneously broadened medium, can generate substantial excess noise at low frequencies through a renormalization of the weak mode relaxation resonance as a result of the intermode coupling. ${ }^{64}$

Another candidate to explain the excess noise measured in some experiments has been optical feedback. ${ }^{56}$ While we believe optical feedback can lead to mode instabilities and therefore indirectly affect the amplitude noise, we have found that optical feedback can be used to actually enhance the squeezing ${ }^{12}$ and therefore is probably not an important source of excess noise.

\subsection{Correlation schemes and squeezing enhancement}

Several schemes have been proposed to enhance the squeezing in pump-suppressed semiconductor lasers. These include amplitude-phase decorrelation, ${ }^{65,11}$ junction voltage feedforward, ${ }^{11}$ injection locking ${ }^{60}$ and optical feedback. ${ }^{66,61,12}$ The method of injection locking seeks to eliminate the excess noise caused by multi-mode operation by selectively reducing the facet loss of one particular mode. In one experiment, ${ }^{60}$ a dye laser was used to injection lock a commercial quantum-well laser, suppressing the side-mode power by more than $10 \mathrm{~dB}$ and reducing the amplitude noise from $1 \mathrm{~dB}$ below the SQL to more than $3 \mathrm{~dB}$ below it. 
The three other schemes take advantage of the residual correlations which occur between the field amplitude, phase and the carrier density at moderate injection currents. Using optical feedback, the phase-amplitude correlation [see equation (4)] can be used to reduce the amplitude noise at injection currents near the onset of squeezing around $R=1$. In this regime, the amplitude noise is close to the shot noise limit and the phase-amplitude correlation [calculated using (4)] is about 0.5 and hence a reduction in the noise by a factor of two is expected. This range of injection currents is potentially important for the generation of squeezed light from room-temperature semiconductor lasers. Due to the danger of thermal damage to the laser facet, most room temperature semiconductor lasers cannot be pumped by more than a few times the threshold current which necessarily limits the squeezing due to the incomplete suppression of the dipole moment and vacuum fluctuations and also due to noise from spontaneous emission into non-lasing modes. Optical feedback could play a role in enhancing the squeezing from such lasers. Recent experimental results ${ }^{61,12}$ have shown the effectiveness of optical feedback in the generation of amplitude squeezed light from a room-temperature semiconductor laser. Optical feedback can also be used to substantially reduce the classical noise in a laser close to threshold ${ }^{5,8}$ where the amplitude-phase correlation is high.

Amplitude-phase decorrelation is a noise reduction technique in which the output field of the laser is sent through an interferometer which translates phase noise into amplitude noise. This results in a simultaneous decorrelation of the field amplitude and phase and reduction of the amplitude noise. Reduction of the classical noise in lasers close to threshold by more than $10 \mathrm{~dB}$ has been demonstrated experimentally by Newkirk and Vahala. ${ }^{67}$ This method can be used to enhance the squeezing of semiconductor lasers. ${ }^{11}$ The final method of amplitude noise reduction mentioned here is feedforward of the junction voltage fluctuations. The correlation between junction voltage and field amplitude is exactly equal in magnitude to the amplitude-phase correlation, (4), in the limit $\alpha \rightarrow \infty$. By feeding the junction voltage signal forward to an intensity modulator, the amplitude noise can be reduced by a factor of 2 when $R=2$.

\section{ULTIMATE STABILITY PROSPECTS}

Using the techniques described above, it is clear that the amplitude and phase noise can be reduced signifcantly. The question that naturally arises is how far these reductions can be pushed. The ultimate limits to the noise reduction are determined by the requirement that the field satisfy the spectral uncertainty relation

$$
P_{\Delta r}(\Omega) P_{\Delta \psi}(\Omega) \geq \frac{1}{4 r_{0}^{2}}
$$

at all frequencies where $r_{0}^{2}$ is the output photon flux in photons/second. Equation (18) is a direct result of the Heisenberg Uncertainty Principle which limits the precision with which two non-commuting variables such as the amplitude and phase of the optical field can be measured.

In a solitary semiconductor laser, phase diffusion produces a phase noise power spectrum which varies inversely with the square of the measurement frequency $\Omega$ below the cavity bandwidth as shown in Figure 11 . Above the cavity bandwidth, the vacuum fluctuations reflected from the front facet of the laser generate a constant phase noise power. Under ideal pump-suppressed conditions with perfect efficiency, the amplitude noise below the cavity bandwidth is found to be proportional to $\Omega^{2}$ resulting in a spectral uncertainty product exactly twice the minimum value given by the equality condition in (18). For Poissonian pumping, the amplitude noise just sits at the SQL at all frequencies and the spectral uncertainty is far greater than the minimum value.

If, for example, optical feedback is applied to pump suppressed laser, it was stated in Section 2.3.1 that in the semiclassical limit, the frequency noise (and therefore the phase noise) can be reduced by a factor of $\left(1+C_{i}+\alpha C_{r}\right)^{2}$ and can in fact be made arbitrarily small if $C$ is made large enough. A fully quantum mechanical analysis of the effects of optical feedback on the laser noise properties has recently been made. ${ }^{24}$ It is found that when optical feedback is applied to a pump-suppressed laser, the phase noise power is still reduced by the amount predicted 


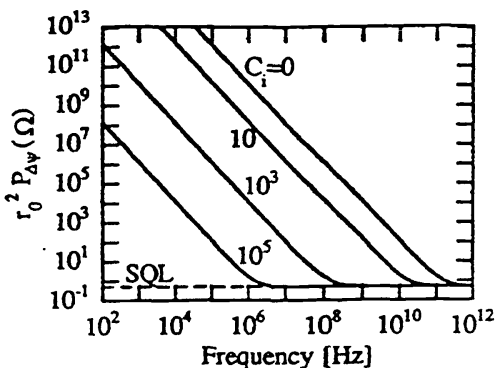

(a)

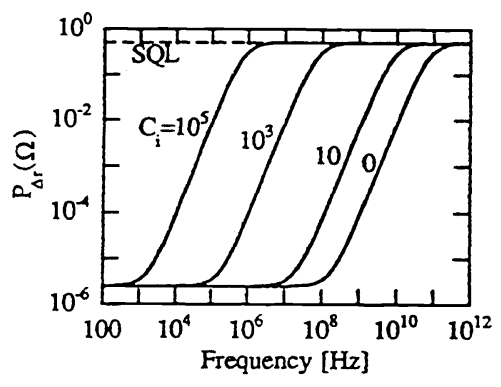

(b)

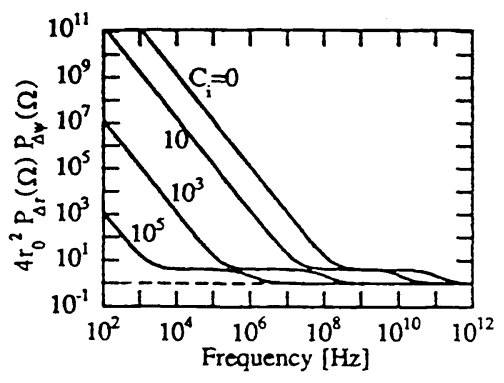

(c)

Figure 11: (a) Phase noise, (b) amplitude noise and (c) spectral uncertainty product in a pump-suppressed semiconductor laser relative the the SQL for different feedback levels $C_{i}$.

semiclassically but the squeezing bandwidth is also reduced to compensate for the additional precision acquired in the measurement of the field phase. This behaviour is shown also in Figure 11 which plots the phase and amplitude noise of the laser for different values of $C_{i}$. Thus, in order to acheive additional frequency stability, the amplitude squeezing must be degraded.

This limitation is not likely to be reached soon, however. If one stipulates a requirement of $10 \mathrm{~dB}$ of amplitude squeezing at $10 \mathrm{MHz}$, then the minimum linewidth which can be obtained by uniform reduction of a white frequency noise spectrum with optical (or electronic) feedback before the spectral uncertainty product starts to degrade the squeezing is about $2 \mathrm{mHz}$. We clearly have a long road to travel before the truly fundamental limits to semiconductor laser noise are acheived.

\section{CONCLUSION}

In this paper, the fundamental limits to noise and its reduction in semiconductor lasers have been discussed. The laser linewidth is determined primarily by noise due to spontaneous emission into the lasing mode. This causes the field phase to diffuse in time thereby broadening the linewidth. Phase-amplitude coupling enhances the linewidth by a large factor by coupling amplitude noise into the phase but also generates a strong correlation between the amplitude and phase at moderate pump rates. Methods of linewidth reduction such as optical feedback take advantage of this correlation in order to reduce the linewidth far below the original SchawlowTownes value. Electronic feedback has also been used extensively to reduce the low-frequency phase noise and generate ultra-narrow linewidths from semiconductor lasers.

The quantum theory of amplitude noise in semicondutor lasers was then discussed and the contributions of the various noise sources elucidated. Pump noise is responsible for producing the SQL at high pump rates but can be suppressed by driving the laser with a constant current source leading the the generation of amplitude squeezed light. Experimental issues involved in the production of amplitude squeezed light from semiconductor lasers are outlined and several ways of enhancing the squeezing are mentioned. Finally, the ultimate limit to simlutaneous amplitude and frequency noise reduction using any technique is established.

\section{REFERENCES}

[1] A. L. Schawlow and C. H. Townes, "Infrared and optical masers," Phys. Rev., vol. 112, pp. 1940-1949, 1958. 
[2] J. P. Gordon, H. J. Zeigler, and C. H. Townes, "The maser - new type of microwave amplifier, frequency standard and spectrometer," Phys. Rev., vol. 99, pp. 1264-1274, 1955.

[3] C. H. Henry, "Theory of the linewidth of semiconductor lasers," IEEE J. Quantum Electron., vol. QE-18, pp. 259-264, 1982.

[4] K. Vahala and A. Yariv, "Semiclassical theory of noise in semiconductor lasers - part I and II," IEEE J. Quantum Electron., vol. QE-19, pp. 1096-1109, 1983.

[5] B. Dahmani, L. Hollberg, and R. Drullinger, "Frequency stabilization of semiconductor lasers by resonant optical feedback," Opt. Lett., vol. 12, pp. 876-878, 1987.

[6] Y. Shevy, J. Iannelli, J. Kitching, and A. Yariv, "Self-quenching of the semiconductor laser linewidth below the Schawlow-Townes limit by using optical feedback," Opt. Lett., vol. 17, pp. 661-663, 1992.

[7] A. Yariv, R. Nabiev, and K. Vahala, "Self-quenching of the fundamental phase and amplitude noise in semiconductor lasers with dispersive loss," Opt. Lett., vol. 15, pp. 1359-1361, 1990.

[8] J. Kitching, R. Boyd, A. Yariv, and Y. Shevy, "Amplitude noise reduction in semiconductor lasers with weak, dispersive optical feedback," Opt. Lett., vol. 19, pp. 1331-1333, 1994.

[9] H. Haug, "Quantum-mechanical rate equations for semiconductor lasers," Phys. Rev., vol. 184, pp. 338-348, 1969.

[10] Y. Yamamot.o, S. Machida, and O. Nilsson, "Amplitude squeezing in a pump-noise-suppressed laser oscillator," Phys. Rev. A, vol. 34, pp. 4025-4041, 1986.

[11] A. Karlsson and G. Björk, "Use of quantum-noise correlation for noise reduction in semiconductor lasers," Phys. Rev. A, vol. 44, pp. 7669-7683, 1991.

[12] J. Kitching, A. Yariv, and Y. Shevy, "Generation of amplitude squeezed light from a room-temperature semiconductor laser with dispersive optical feedback,". Submitted to Phys. Rev. Lett.

[13] C. E. Weiman and L. Hollberg, "Using diode lasers for atomic physics," Rev. Sci. Instrum., vol. 62, pp. 1-20, 1991.

[14] A. S. Zibrov, R. W. Fox, R. Ellingsen, C. S. Weimer, V. L. Velichansky, G. M. Tino, and L. Hollberg, "High-resolution diode-laser spectroscopy of calcium," Appl. Phys. B, vol. 59, pp. 327-331, 1994.

[15] M. T. Jaekel and S. Reynaud, "Quantum limits to interferometric measurements," Europhys. Lett., vol. 13, pp. 301-306, 1990.

[16] B. E. A. Saleh and M. C. Teich, "Information transmission with photon-number-squeezed light," Proc. IEEE, vol. 80, pp. 451-460, 1992.

[17] J. G. Rarity and P. R. Tapster, "Quantum communications," Appl. Phys. B, vol. 55, pp. 298-303, 1992.

[18] M. Lax, "Classical noise V. Noise in self-sustained oscillators," Phys. Rev., vol. 160, pp. 290-307, 1967.

[19] A. Yariv, Optical Electronics. Philadelphia: Saunders College Pub., 1991.

[20] A. Yariv, Quantum Electronics. New York: John Wiley and Sons, 1987.

[21] M. W. Flemming and A. Mooradian, "Fundamental line broadening of single-mode (GaAl)As diode lasers," Appl. Phys. Lett., vol. 38, pp. 511-513, 1981.

[22] C. Harder, K. Vahala, and A. Yariv, "Measurement of the linewidth enhancement factor $\alpha$ of semiconductor lasers," Appl. Phys. Lett., vol. 42, pp. 328-330, 1983.

[23] N. Ogasawara, R. Ito, and R. Morita, "Linewidth enhancement factor in GaAs/AlGaAs multi-quantum-well lasers," Jap.J. Appl. Phys., vol. 24, pp. L519-L521, 1985. 
[24] J. Kitching, Y. Shevy, and A. Yariv, "Quantum mechanical analysis of amplitude and phase noise in a semiconductor laser with optical feedback,". To be published.

[25] P. H. Laurent, A. Clairon, and C. H. Bréant, "Frequency noise analysis of optically self-locked diode lasers," IEEE J. Quantum Electron., vol. 25, pp. 1131-1142, 1989.

[26] Y. Shevy, J. Kitching, and A. Yariv, "Linewidth reduction and frequency stabilization of a semiconductor laser with a combination of FM sideband locking and optical feedback," Opt. Lett., vol. 18, pp. 1071-1073, 1993.

[27] R. Wyatt and W. J. Devlin, "10 kHz linewidth $1.5 \mu \mathrm{m}$ InGaAsP external cavity laser with $55 \mathrm{~nm}$ tuning range," Electron. Lett., vol. 19, pp. 110-112, 1983.

[28] E. Pat.?ak, A. Sugimura, S. Saito, T. Mukai, and H. Oleson, "Semiconductor laser linewidth in optical feedback configurations," Electron. Lett., vol. 19, pp. 1026-1027, 1983.

[29] R. Lang and K. Kobayashi, "External optical feedback effects on semiconductor injection laser properties," IEEE J. Quantum Electron., vol. QE-16, pp. 347-355, 1980.

[30] E. Patzak, H. Oleson, A. Sugimura, S. Saito, and T. Mukai, "Spectral linewidth reduction in semiconductor lasers by an external cavity with weak optical feedback," Electron. Lett., vol. 19, pp. 938-940, 1983.

[31] K. Vahala and A. Yariv, "Detuned loading in coupled cavity semiconductor lasers - effect on quantum noise and dynamics," Appl. Phys. Lett., vol. 45, pp. 501-503, 1984.

[32] P. Spano, S. Piazzolla, and M. Tamburrini, "Theory of noise in semiconductor lasers in the presence of optical feedback," IEEE J. Quantum. Electron., vol. QE-20, pp. 350-357, 1984.

[33] H. Li and N. B. Abraham, "Analysis of the noise spectra of a laser diode with optical feedback from a high-finesse resonator," IEEE J. Quantum Electron., vol. 25, pp. 1782-1793, 1989.

[34] D. R. Hjelme, A. R. Mickelson, and R. G. Beausoleil, "Semiconductor laser stabilization by external optical feedback," IEEE J. Quantum Electron., vol. 2T, pp. 352-372, 1991.

[35] G. P. Agarwal, "Line narrowing in a single-mode injection laser due to external optical feedback," IEEE J. Quantum Electron., vol. QE-20, pp. 468-471, 1984.

[36] J. M. Iannelli, Y. Shevy, J. Kitching, and A. Yariv, "Linewidth reduction and frequency stabilization of semiconductor lasers using dispersive losses in an atomic vapor," IEEE J. Quantum Electron., vol. 29, pp. 1253-1261, 1993.

[37] F. Favre, D. L. Guen, and J. C. Simon, "Optical feedback effects upon laser diode oscillation field spectrum," IEEE J. Quantum Electron., vol. QE-18, pp. 1712-1717, 1982.

[38] W. D. Lee and J. C. Campbell, "Narrow-linewidth frequency stabilized $A l_{x} G a_{1-x} A s / G a A s$ laser," $A p p l$. Phys. Lett., vol. 60, pp. 1544-1546, 1992.

[39] J. Kitching, Y. Shevy, J. Iannelli, and A. Yariv, "Measurements of $1 / \mathrm{f}$ frequency noise reduction in semiconductor lasers using optical feedback with dispersive loss," J.' Lightwave Tech., vol. 11, pp. 1526-1532, 1993.

[40] D. Halford, "Infra-red microwave frequency synthesis design: some relevant conceptual noise aspects," in Proc. Frequency Standards and Metrology Seminar, (Canada), pp. 431-466, 1971.

[41] R. V. Pound, "Electronic frequency stabilization of microwave oscillators," Rev. Sci. Instrum., vol. 17, pp. 490-505, 1946.

[42] R. W. P. Drever, J. L. Hall, F. V. Kowalski, J. Hough, G. M. Ford, A. J. Munley, and H. Ward, "Laser phase and frequency stabilization using an optical resonator," Appl. Phys. B, vol. 31, pp. 97-105, 1983. 
[43] C. Shin and M. Ohtsu, "Stable semiconductor laser with a 7-hz linewidth by and optical-electronic doublefeedback technique," Opt. Lett., vol. 15, pp. 1455-1457, 1990.

[44] K. Nakagawa, M. Kourogi, and M. Ohtsu, "Frequency noise reduction of a diode laser by using the fm sideband technique," Opt. Lett., vol. 17, pp. 934-936, 1992.

[45] D. E. McCumber, "Intensity fluctuations in the output of cw laser oscillators. I," Phys. Rev., vol. 141, pp. 306-322, 1966.

[46] M. Lax, "Quantum noise VII: the rate equations and amplitude noise in lasers," IEEE J. Quantum Electron., vol. QE-3, pp. 37-46, 1967.

[47] C. M. Caves, "Quantum mechanical noise in an interferometer," Phys. Rev. D, vol. 23, pp. 1693-1708, 1981.

[48] Y. Yamamoto and S. Machida, "High-impedance suppression of pump fluctuation and amplitude squeezing in semiconductor lasers," Phys. Rev. A, vol. 35, pp. 5114-5130, 1987.

[49] M. Lax and W. H. Louiselle, "Quantum noise IX: quantum Fokker-Planck solution for laser noise," IEEE J. Quantum Electron., vol. QE-3, pp. 47-58, 1967.

[50] M. Sargent III, M. O. Scully, and W. E. Lamb Jr., Laser Physics. Reading, Massachusetts: Addison-Wesley, 1974.

[51] L. Wu, H. J. Kimble, J. L. Hall, and H. Wu, "Generation of squeezed states by parametric down conversion," Phys. Rev. Lett., vol. 57, pp. 2520-2523, 1986.

[52] R. E. Slusher, L. W. Hollberg, B. Yurke, J. C. Mertz, and J. F. Valley, "Observation of squeezed states generated by four wave mixing in an optical cavity," Phys. Rev. Lett., vol. 55, pp. 2409-2412, 1985.

[53] S. Machida and Y. Yamamoto, "Ultrabroadband amplitude squeezing in a semiconductor laser," Phys. Rev. Let.., vol. 60, pp. 792-794, 1988.

[54] S. Machida, Y. Yamamoto, and Y. Itaya, "Observation of amplitude squeezing in a constant-current-driven semiconductor laser," Phys. Rev. Lett., vol. 58, pp. 1000-1003, 1987.

[55] S. Machida and Y. Yamamoto, "Observation of amplitude squeezing from semiconductor lasers by balanced direct detectors with a delay line," Opt. Lett., vol. 14, pp. 1045-1047, 1989.

[56] W. H. Richardson and R. M. Shelby, "Nonclassical light from a semiconductor laser operating at 4K," Phys. Rev. Lett., vol. 64, pp. 400-403, 1990.

[57] W. H. Richardson, S. Machida, and Y. Yamamoto, "Squeezed photon-number noise and sub-poissonian electrical partition noise in a semiconductor laser," Phys. Rev. Lett., vol. 66, pp. 2867-2870, 1991.

[58] H. A. Bachor, P. Rottengatter, and C. M. Savage, "Correlation effects in light sources with high quantum efficiency," Appl. Phys. B, vol. 55, pp. 258-264, 1992.

[59] M. J. Freeman, H. Wang, D. G. Steel, R. Craig, and D. Scifres, "Amplitude-squeezed light from quantum well lasers," Opt. Lett., vol. 18, pp. 379-381, 1993.

[60] H. Wang, M. J. Freeman, and D. G. Steel, "Squeezed light from injection-locked quantum well lasers," Phys. Rev. Lett., vol. 71, pp. 3951-3954, 1993.

[61] M. J. Freeman, H. Wang, D. G. Steel, R. Craig, and D. R. Scifres, "Wavelength-tunable amplitude-squeezed light from a room-temperature quantum well laser," Opt. Let., vol. 18, pp. 2141-2143, 1993.

[62] P. R. Tapster, J. G. Rarity, and J. S. Satchell, "Generation of sub-poissonian light by high-efficiency lightemitting diodes," Europhys. Lett., vol. 4, pp. 293-299, 1987. 
[63] S. Machida and Y. Yamamoto, "Quantum limited operation of balanced mixer homodyne and heterodyne receivers," IEEE J. Quantum Electron., vol. QE-22, pp. 617-624, 1986.

[64] C. B. Su, J. Schlafer, and R. B. Lauer, "Explanation of low-frequency relative intensity noise in semiconductor lasers," Appl. Phys. Lett., vol. 57, pp. 849-851, 1990.

[65] M. A. Newkirk and K. J. Vahala, "Amplitude-phase decorrelation: a method for reducing intensity noise in semiconductor lasers," J. Quantum Electron., vol. 27, pp. 13-22, 1991.

[66] J. Kitching, Y. Shevy, and A. Yariv, "Quantum limits to amplitude noise reduction in semiconductor lasers with weak optical feedback and dispersive loss," in Proceedings of the annual meeting of the Optical Society of America, 1993.

[67] M. A. Newkirk and K. J. Vahala, "Large $(14.5 \mathrm{~dB})$ reduction of intensity noise from a semiconductor laser by amplitude-phase decorrelation," Appl. Phys. Lett., vol. 60, pp. 1289-1291, 1992. 